\title{
Evaluation and Simulation of An Industrial Sonoreactor Performance
}

\author{
R.R. Gabriela1 ${ }^{1} 2^{*}$, G.T. Claudia Del Carmen ${ }^{1}$, J.B. Jose Alfredo ${ }^{1}$, A.G. Alejandro 3 , G.N. Israel ${ }^{4}$, M.D. Sergio Alejandro ${ }^{5}$ \\ ${ }^{1}$ National Polytechnic Institute, SEPI-ESIME Zacatenco, U.P. Adolfo López Mateos, Lindavista, CP 07738, CDMX, México. \\ ${ }^{2}$ Istmo University, University City, S/N, Barrio Santa Cruz 4 Secc., Sto. Domingo Tehuantepec, Oaxaca, CP 70760, México. \\ ${ }^{3}$ Engineering and Industrial Development Center, CONACyT-, Carretera Champotón-Cd. del Carmen 408, Cd. del Carmen, Campeche, 24150 México. \\ ${ }^{4}$ Process Engineering Postgraduate, Metropolitan Autonomous University, Azcapotzalco, Av. San Pablo 180. Azcapotzalco, 07740 México D.F., México. \\ ${ }_{5}^{5}$ Basic Sciences Department, Metropolitan Autonomous University, Azcapotzalco, Av. San Pablo 180. Azcapotzalco, 07740 México D.F., México.
}

\section{A R T I C LEDET A I LS}

Article history:

Received 28 October 2019

Accepted 11 November 2019

Available online 10 December 2019

Keywords:

Industrial Sonoreactor

LES

Axial Flow Number

PIV

\begin{abstract}
A B S T R A C T
In this work, the velocity fields and vorticity of an industrial bath sonoreactor of $500 \mathrm{kHz}$ were obtained experimentally evaluated by PIV technique. Based on the results obtained immediately after the sonoreactor was turned on, the velocity and the axial flow number $\left(N_{Q \text { ax (z) }}\right)$, were calculated at different times. The formation of stable vortexes, near the sonoreactor walls, were observed after 5 minutes of sonoreactor operation. Due to the difficulty of perform measurements during the initial operation time simulations performed at small period of time were performed by the Large Eddy Simulation (LES), showing the trend of the flow in the sonoreactor.
\end{abstract}

\section{Introduction}

Ultrasound is a new advanced technology and it is used to intensify several physical and chemical processes through the generation of cavitation and acoustic currents in liquid medium. It has been successfully used in liquid medium, for example, for the removal of contaminating dyes from wastewater [1], in chemical reactions [2], water treatment [3], heat transfer surfaces [4] desorption [5], cleaning [6], crystallization [7, 8], among other applications. In comparison with other technologies, the use of ultrasonic energy saves and optimizes energy consumption, improving product quality and reducing production time [9]. The potential of power ultrasound involves physical and chemical processes. Physical processes are mainly ascribed to mechanical effects of the high-intensity waves in any medium while chemical processes refer to chemical effects induced by ultrasonic cavitation in liquids. The latter processes are included in the term of sonochemistry. The general term for the whole area is sonoprocessing or ultrasonic processing [10]. Several authors have studied the effect of ultrasound on fluid properties, having different configurations regarding the reactors used. Some of the simulation studies using CFD in sonochemical reactors include the research carried out for evaluating the mixing rate modeling the piezoelectric movement at a frequency of $1.7 \mathrm{MHz}$ [11], which found the acoustic current was in the direction of the propagation of the wave and its maximum velocity near the piezoelectric surface. Another work [12] investigated the effect of ultrasonic waves (microcurrents and cavitation) of $24 \mathrm{kHz}$ and $1.7 \mathrm{MHz}$ on the heat transfer of a thin platinum wire. It is important to emphasize that the most studies about ultrasonic reactors has been carried out on horn laboratory reactors, in this sense it has been studied the numerical simulation of ultrasonic horn transducer (acoustic supercavitation) [13] and tested various cavitation models, reporting that none of them adequately predicted the main characteristics of the cavitation flow in the vicinity of the sonotrode tip. Moreover, it was investigated the influence of acoustic current induced by low frequency ultrasound on mass transfer in a two-phase 3D system [14], for this purpose it was compared the operation of two different sonoreactors with a stirred tank reactor; it was found that the volumetric mass transfer coefficient presents a linear relationship (with the slope of +1 ) with the ultrasound amplitude. As a

consequence of the phenomena related to ultrasound, there are important parameters to analyze in a reactor whose determination is the key to understanding mixing, which in turn affects the efficiency of chemical reactions, such as circulation number, which have been studied mainly in stirred tank reactors [15]. However, there is not experimental studies that report the axial flow number $\left(\mathrm{N}_{\mathrm{Qax}(\mathrm{z})}\right)$ or the circulation number in industrial sonoreactors. More research on flow and simulation parameters, as well as operational parameters such as the flow number, will lead to a better understanding of ultrasonic reactors, which will make it possible to scale up them, which It represents a challenge today.

\section{Experimental Methods}

\subsection{PIV Measurements}

It was used an industrial sonoreactor of $500 \mathrm{kHz}$ and a maximum power of 500 watts. In the test $60 \%$ of the maximum power was used. The sonoreactor used was provided of plexiglas walls to visualize its performance and has 6 piezoelectrics of $1.5 \mathrm{~cm}$ diameter (D) located as shown in Fig. 1. The fluid used was distilled water with the following conditions; $25^{\circ} \mathrm{C}, \rho=998.2 \mathrm{~kg} / \mathrm{m}^{3}, \mu=1.003 \mathrm{mPas}$, and $10 \mathrm{~cm}$ of liquid height $(\mathrm{H})$ were used in all tests. The particle image velocimetry (PIV) was performed by Quantel EverGreen $®$ laser, with a laser pulse with less than or equal to 10 nanoseconds at nominal energy. It has a 4MP-LS camera, Model 630090 PowerView $^{\mathrm{TM}}$, with a resolution of 2360 x 1776 pixels. Hollow glass spheres with silver coat of approximate $10 \mu \mathrm{m}$ diameter and density $\rho=1020 \mathrm{~kg} / \mathrm{m}^{3}$ were used as seeding particles. During the tests, the camera was approximately $70 \mathrm{~cm}$ from the analysis plane, located at the middle length $(12.5 \mathrm{~cm})$ of the sonoreactor.

The height of the PIV measurements plane was adjusted to avoid the laser reflection, from the stainless-steel bottom and the liquid surface because it causes interference with measurements. Therefore, the measurements were taken $1.2 \mathrm{~cm}$ from the bottom and $1.0 \mathrm{~cm}$ from the liquid surface, then the pictures were taken every $\Delta t=3500 \mu \mathrm{s}$ and the capture rate was fixed to 7.25 frames/s.

The TSI Insight 4G software was applied to analyze the pictures by applying the Nyquist recursive grid algorithm, with an interrogation window of $32 \times 32$ pixels and a $50 \%$ of overlap. The velocity fields were obtained from the average of 350 pairs of images taken at the analysis plane (Fig. 1). 


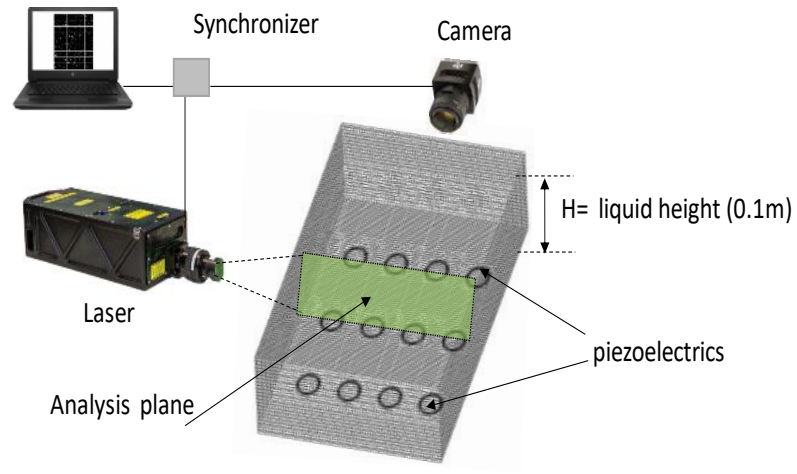

Sonoreactor

Fig. 1 PIV system to evaluate the performance of the sonoreactor

\subsection{Performance of the Sonoreactor as a Function of Time}

To evaluate the velocity fields of the reactor immediately after the turn on the reactor, the PIV was turned on at the same time as the sonoreactor and the measurements of velocity were carried out every $1 / 15 \mathrm{~s}$ for $20 \mathrm{~s}$. The velocity field was evaluated in each time step.

\subsection{Performance of the Sonoreactor at Steady State}

PIV measurements of the velocity were performed five minutes after the sonoreactor was turned on.

\subsection{Simulations of the Sonoreactor}

The numerical simulations were performed with the commercial software FLUENT V.15.0 and was used the Large Eddy Simulation (LES), with Smagorinsky-Lilly subrgrid scale model. The mesh has 1662611 tetrahedral elements (Fig. 2). The piezoelectric were defined as velocity inlet $\left(\mathrm{v}_{0}(\mathrm{t})\right)$, defining a UDF as the one shown in Eq.(1) [16]. The time step was $1 \times 10^{-7} \mathrm{~s}$

$$
\begin{array}{ll} 
& \mathrm{v}_{0}(\mathrm{t})=\omega \mathrm{A}_{0} \sin (\omega \mathrm{t}) \\
\text { and } & \mathrm{w}=2 \pi f
\end{array}
$$

where: $\mathrm{A}_{0}=$ maximum piezoelectric displacement $(\mathrm{m}), \mathrm{t}=$ time $(\mathrm{s}), f=$ frequency $(1 / \mathrm{s})$.

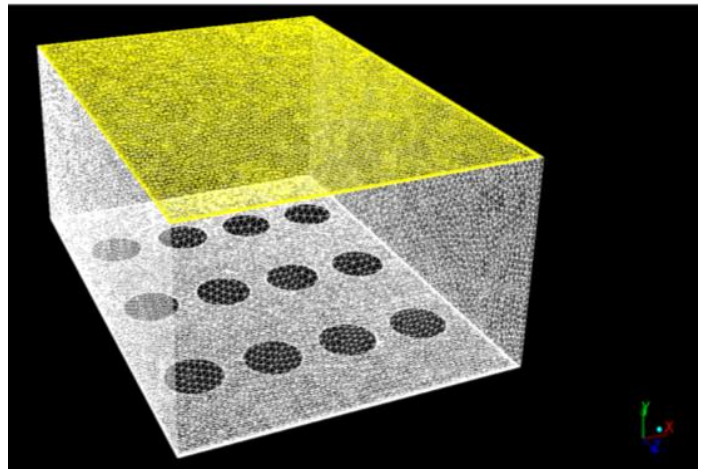

Fig. 2 Sonoreactor mesh used wiht the Large Eddy Simulations (LES)

\section{Results and Discussion}

\subsection{Performance of the Sonoreactor as a Function of Time}

Fig. 3 shows the variation of the mean velocity magnitude at different liquid heights $(\mathrm{H})$ and time. As can be seen the highest velocities are reached at a liquid height between $0.030 \mathrm{~m}$ to $0.55 \mathrm{~m}$ (doted rectangle)

Based on the velocity and the sonoreactor area, the average axial flow rate $\left(Q_{a x(z)}\right)$ was calculated and was used to evaluate the axial flow number $\left(N_{\operatorname{Qax}(z)}\right)$, similar to the one of used in impellers [17], which was defined for sonoreactors as in Eq.(3).

$$
N_{Q a x(z)}=\frac{Q \operatorname{ax}(z)}{f d_{t}{ }^{3}}
$$

Fig. 4 shows the $N_{Q \operatorname{ax}(z)}$ at different liquid heights $(\mathrm{H})$ as at different time of sonoreactor operation. As it is shown, the highest $N_{\text {Qax (z) }}$ are produced at $\mathrm{H}$ between $0.030 \mathrm{~m}$ to $0.55 \mathrm{~m}$, because in this zone the highest velocities are reached (Fig. 4).

https://doi.org/10.30799/jacs.215.19050401
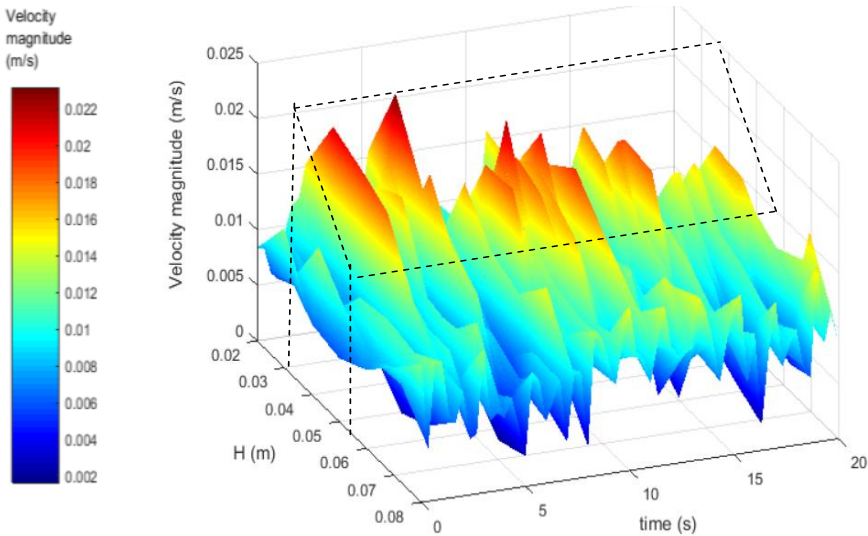

Fig. 3 Variation of the mean velocity magnitude at different liquid heights and time
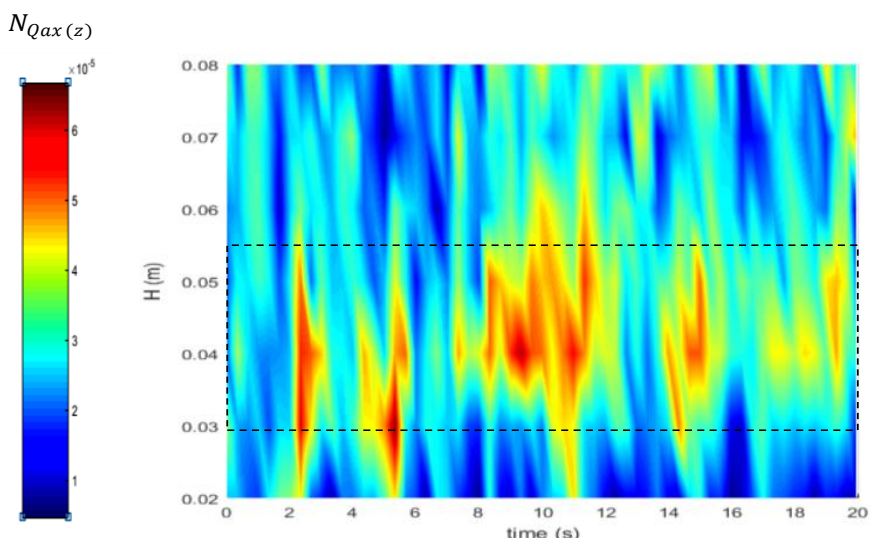

Fig. 4 Variation of the axial flow number $\left(N_{\text {Qax }(z)}\right)$ at different liquid heights $(\mathrm{H})$ and time

\subsection{Performance of the Sonoreactor at Steady State}

After five minutes of sonoreactor operation, the velocity magnitude was evaluated by PIV. Fig. 5 shows that higher zones of high velocities were obtained than those obtained during the first $20 \mathrm{~s}$ of operation (Fig. 3). This is because after 5 minutes of operation the system reached the steady state. As it is shown, there are two important zones of high velocity (in red), and two important vortexes near the walls of the sonoreactor.

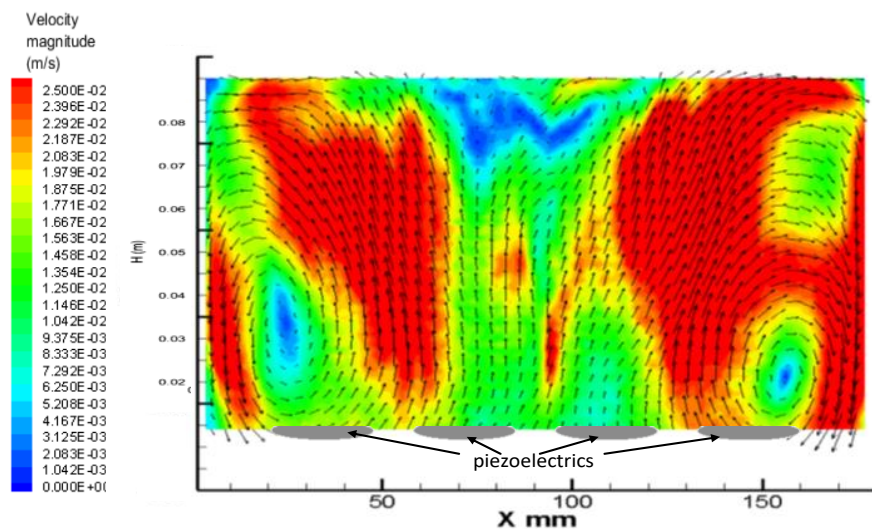

Fig. 5 Velocity magnitude contours obtained by PIV

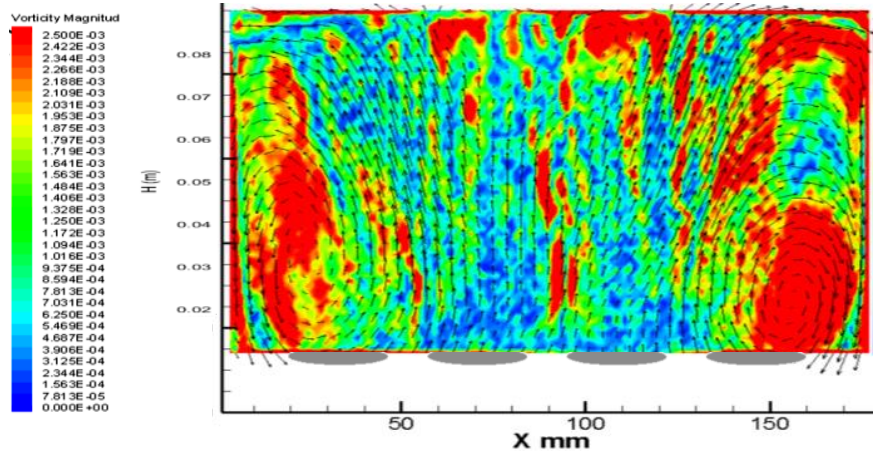

Fig. 6 Vorticity magnitude contours obtained by PIV 
The piezoelectrics at the central part of the sonoreactor dive the flow up, while in the ones near the walls, the flow is diverted due to the descendent flow generating the vortex. In the zones of high velocity, the vorticity also is higher, as it is shown in Fig. 6. In these zones mixing is higher than in the central zone, which improve the mass transfer and the reactions in the sonoreactor.

\subsection{Simulations of the Sonoreactor at the First Operation Times}

Due to the difficulty of measuring the performance of the sonoreactor at the first operation times, simulations were performed. Fig. 7 shows the results of the sonoreactor behavior at $0.01 \mathrm{~s}$. As can be seen, the vortex that are being formed near the walls and at the central part, will become in the largest stable vortex, as shown in Fig. 5. It is important to note, that there are two main jets formed at the piezoelectrics nearest the walls, that in difference with other works [18], disappear with the time, as shown in the experimental results obtained by PIV (Fig. 5). This is because the interaction among the piezoelectrics, in the steady state, produce two great vortices near the walls that vanishes the jets formed immediately after starting up the sonoreactor.
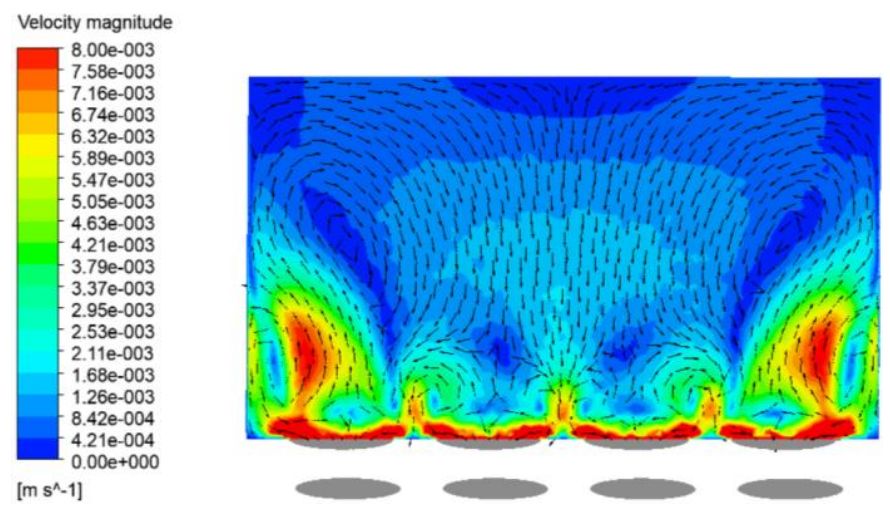

Fig. 7 Velocity magnitude contours obtained by LES

Fig. 8 shows the simulated results of vorticity magnitude. As can be seen, the vorticity is located in the low part at the first times of sonoreactor operation, near the piezoelectrics, however as the fluid gets stability, the higher vorticity near the walls are formed, as shown in Fig. 6. The results, show that flow does not reach stability immediately after the sonoreactor is turned on. The zones of higher velocity and velocity at the first moments are smaller near the piezoelectrics. However, those zones increase after the time and get stability.

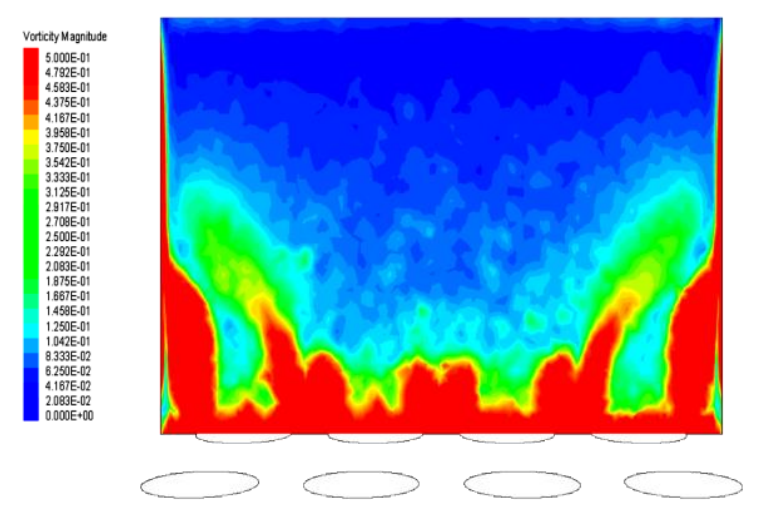

Fig. 8 Vorticity magnitude contours obtained by LES

\section{Conclusion}

The performance of a sonoreactor of $500 \mathrm{kHz}$ was evaluated using PIV at different time operation. The reactor was simulated at the first instants of initiated its operation. The results show that the velocity and vorticity fields at the first times of operation are reduce, but at longer periods of operation increase and trend to stabilize, as it was measured experimentally by PIV. This technique has demonstrated to be an important measurement tool to evaluate the performance of this kind of reactors. The simulation needs to be more extensive using more powerful computers due to the time step to capture the effects at high frequencies is too small, increasing the computational calculation time.

\section{References}

[1] M. May-Lozano, V. Mendoza-Escamilla, E. Rojas-García, R. López-Medina, G. Rivadeneyra-Romero, S.A. Martínez-Delgadillo, Sonophotocatalytic degradation of Orange II dye using low cost photocatalyst, J. Clean. Prod. 148 (2017) 836-844.

[2] Z.L. Wu, J. Lifka, B. Ondruschka, Comparison of energy efficiency of various ultrasonic devices in aquasonochemical reactions, Chem. Eng. Tech. 29 (2006) 610-615.

[3] H. Duckhouse, T.J. Mason, S.S. Phull, J.P. Lorimer, The effect of sonication on microbial disinfection using hypochlorite, Ultrason. Sonochem. 11 (2004) 173176.

[4] M. Legay, B. Simony, P. Boldo, N. Gondrexon, S. Le Person, A. Bontemps, Improvement of heat transfer by means of ultrasound: Application to a doubletube heat exchanger, Ultrason. Sonochem. 19 (2012) 1194-1200.

[5] S. Gantert, D. Möller, Ultrasonic desorption of $\mathrm{CO}_{2}-\mathrm{A}$ new technology to save energy and prevent solvent degradation, Chem. Eng. Tech. 35 (2012) 576-578

[6] A.D. Farmer, A.F. Collings, G.J. Jameson, The application of power ultrasound to the surface cleaning of silica and heavy mineral sands, Ultrason. Sonochem. 7 (2000) 243-247.

[7] K. Seo, S. Suzuki, T. Kinoshita, I. Hirasawa, Effect of ultrasonic irradiation on the crystallization of sodium acetate trihydrate utilized as heat storage material, Chem. Eng. Tech. 33 (2012) 1013-1016.

[8] R. Ambrus, N.N. Amirzadi, P. Sipos, P. Szabo-Revesz, Effect of sonocrystallization on the habit and structure of gemfibrozil crystals, Chem. Eng. Tech. 33 (2010) 827-832.

[9] L. Nastac, Mathematical modeling of the solidification structure evolution in the presence of ultrasonic stirring, Metall. Mater. Trans. B 42 (2011) 1297 1305.

[10] J.A. Gallego-Juárez, High-power ultrasonic processing: recent developments and prospective advances, Phys. Procedia 3 (2010) 35-47.

[11] F. Parvizian, M. Rahimi, N. Azimi, A.A. Alsairafi, CFD modeling of micromixing and velocity distribution in a 1.7-MHz tubular sonoreactor, Chem. Eng. Tech. 37 (2013) 113-122.

[12] M. Dehbani, M. Rahimi, M. Abolhasani, A. Maghsoodi, P.G. Afshar, A.R Dodmantipi, A.A. Alsairafi, CFD modeling of convection heat transfer using 1.7 $\mathrm{MHz}$ and $24 \mathrm{kHz}$ ultrasonic waves: a comparative study, Heat Mass Transfer. 50 (2014) 1319-1333.

[13] A. Znidarcic, R. Mettin, M. Dular, Modeling cavitation in a rapidly changing pressure field - Application to a small ultrasonic horn, Ultrason. Sonochem. 22 (2015) 482-492.

[14] B. Sajjadi, S. Asgharzadehahmadi, P. Asaithambi, A.A. Raman, R. Parthasarathy, Investigation of mass transfer intensification under power ultrasound irradiation using 3D computational simulation: A comparative analysis, Ultrason. Sonochem. 43 (2017) 504-518.

[15] D.B. Holmes, R.M. Vocken, J.A. Dekker, Fluid flow in turbine-stirred, baffled tanks-I: Circulation time, Chem. Eng. Sci. 19 (1964) 201-208.

[16] M. Abolhasani, M. Rahimi, Dehbani, A.A. Alsairafi, CFD modeling of heat transfer by 1.7 MHz ultrasound waves, Numer. Heat Transf. A 62 (2012) 822-841.

[17] M. Alliet-Gaubert, R. Sardeing, C. Xuereb, P. Hobbes, B. Letellier, P. Swaels, CFD analysis of industrial multi-staged stirred vessels, Chem. Eng. Process 45 (2006) 415-427.

[18] B. Sajjadi, A.A.A. Raman, S. Ibrahim, A comparative fluid flow characterisation in a low frequency/high power sonoreactor and mechanical stirred vessel, Ultrason. Sonochem. 27 (2015) 359-373. 